Recepción: 18 / 10 / 2016

Aceptación: 26 / 01 / 2017

Ciencias de la educación

Publicación: 18 / 05 / 2017

Artículo corto

\title{
Algunas especificidades acerca de la Ergonomía y los factores de riesgo en salud ocupacional
}

\author{
Some specificities about Ergonomics and occupational health risk factors
}

Alguns detalhes sobre a ergonomia e fatores de risco em saúde ocupacional

\author{
Mercedes J. Delgado-Carrillo ${ }^{\mathrm{I}}$ \\ mercydc09@hotmail.com \\ Diego J. Cuichán-Nuñez ${ }^{\text {II }}$ \\ jota_tati@hotmail.com \\ Mercy T. Sancán-Moreira ${ }^{\text {II }}$ \\ mercy_sancan@hotmail.ecom
}

Correspondencia: mercydc09@hotmail.com

${ }^{\text {I }}$ Magister en Emergencias Médicas, Doctora en Medicina y Cirugía, Docente de la Universidad Laica Eloy Alfaro de Manabí, Manta, Ecuador.

II Magister en Seguridad y Salud Ocupacional, Licenciado en Terapia Ocupacional, Docente de la Universidad Laica Eloy Alfaro de Manabí, Manta, Ecuador.

III Magister en Gerencia Educativa, Diploma Superior en Educación Universitaria por Competencias, Licenciada en la Especialización de Terapia Ocupacional, Docente de la Universidad Laica Eloy Alfaro de Manabí, Manta, Ecuador. 



\section{Resumen}

La ergonomía es la ciencia que estudia cómo adecuar la relación del ser humano con su entorno, según la definición oficial que el Consejo de la Asociación Internacional de Ergonomía (IEA, por sus siglas en inglés) adoptó en agosto del 2000. Se realizó una revisión bibliográfica en la cual se destaca la definición, la importancia de la visión ergonómica del trabajo, la salud ocupacional, las precauciones a tener en cuenta en la postura, en el sistema visual, en el cardiovascular, así como las correcciones de estas manifestaciones para prevenir enfermedades profesionales. La ergonomía y los factores de riesgo de salud ocupacional deben ser contemplados de forma sistematizada en cada puesto laboral, mediante las revisiones.

Palabras clave: ergonomía; salud ocupacional; factores de riesgo.

\section{Abstract}

Ergonomics is the science that studies how to adapt the relationship between the human being and his environment, according to the official definition adopted by the Council of the International Association of Ergonomics (IEA) in August 2000. A review was carried out Bibliographical in which the definition, the importance of the ergonomic vision of the work, the occupational health, the precautions to be taken into account in the posture, in the visual system, in the cardiovascular system, as well as the corrections of these manifestations to prevent Occupational diseases. Ergonomics and occupational health risk factors should be systematically considered in each job post, through reviews.

Keywords: ergonomics; occupational health; risk factor's.

\section{Resumo}

A ergonomia é a ciência que estuda como ajustar a relação entre os seres humanos e seu meio ambiente, de acordo com a definição oficial pelo Conselho da Associação Internacional de Ergonomia (IEA, por sua sigla em Inglês), adoptado em Agosto de 2000. Foi realizada uma revisão literatura em que a definição enfatiza a importância da visão ergonomia do trabalho, saúde ocupacional, as precauções a tomar em consideração a posição no sistema visual, cardiovascular e correções dessas manifestações para impedir doenças profissionais. Ergonomia e 
fatores de risco ocupacionais para a saúde devem ser cobertos de forma sistemática em cada posto de trabalho através de revisões.

Palavras chave: ergonomia; saúde ocupacional; fatores de risco.

\section{Introducción}

Se ha escrito mucho sobre el origen y la historia de la Ergonomía. Con la única intención de reconocer momentos importantes en el desarrollo de esta especialidad, mencionaremos algunos aspectos relacionados con su devenir histórico. El hombre primitivo se esforzó notablemente por fabricar instrumentos de trabajo y de guerras cada vez más efectivas y fáciles de manejar.

Con un criterio de máxima amplitud, puede considerarse que la Ergonomía nació con la actividad humana, pensada y dirigida a metas. Hace más de 2500 años antes de enunciarse el concepto de ergonomía, parece que sus principios básicos se utilizaron en la Grecia antigua, de los que quedaron como póstumo homenaje sus utensilios, la forma en que concibieron sus teatros y las ventajas que brindaban a sus venerados ancianos. (Almirall Hernández J 2014).

Apud, E, Meyer, F. (2003) plantean en su investigación que el término ergonomía deriva de dos palabras griegas: ergo (trabajo) y nomos (leyes, reglas). Por lo tanto, en el estricto sentido de la palabra, significa leyes o reglas del trabajo. Fue introducida en 1949 por el psicólogo británico K.F.H. Murrell, cuando un grupo de científicos se reunió en Inglaterra para formar la Sociedad de Investigaciones Ergonómicas. La idea fue cobijar bajo el mismo alero a ingenieros, fisiólogos, anatomistas, psicólogos, higienistas industriales, arquitectos, profesionales del área de la salud y en general personas interesadas en el comportamiento humano en el trabajo. Murrell (1969), en su clásico libro denominado Ergonomics, señala la razón que lo llevó a proponer la palabra ergonomía. Él plantea que es simple, que se puede traducir a cualquier idioma y, lo más importante, que no otorga preponderancia a ninguna especialidad en particular, lo que resalta su carácter multidisciplinario.

La Sociedad de Ergonomía define esta disciplina como el estudio científico del hombre en su trabajo; en particular la aplicación de conceptos de anatomía, fisiología y psicología humanas en el diseño del trabajo. Con el correr del tiempo, algunos especialistas en el tema han considerado que esta definición es restrictiva y han propuesto otras más de acuerdo con su carácter 
interdisciplinario. Entre ellos, Zander (1986) señala que "la ergonomía es el estudio del hombre en el trabajo, con el propósito de lograr un óptimo sistema hombre-tarea, en el cual pueda mantenerse un adecuado balance entre el trabajador y las condiciones laborales". En otras palabras, la ergonomía es una disciplina que actúa como un puente entre la biología humana y la ingeniería, poniendo a disposición de ésta última conocimientos de las capacidades y limitaciones humanas que deben ser utilizados para un buen diseño del trabajo. (Apud, E, Meyer, F. 2003).

\section{Importancia de la visión ergonómica del trabajo}

Los objetivos de la ergonomía son promover la salud y el bienestar, reducir los accidentes y mejorar la productividad de las empresas. Al observar los objetivos enumerados, cabe la pregunta: ¿aporta algo nuevo la ergonomía? No hay duda que la seguridad industrial al igual que la medicina del trabajo se ha preocupado de la salud y la prevención de accidentes, alcanzando un alto grado de desarrollo en este siglo. Lo mismo puede afirmarse con relación a la psicología del trabajo, cuyos métodos están orientados a fomentar el bienestar de los trabajadores. Por otra parte, con relación a la eficiencia, desde la época de la revolución industrial hasta el actual desarrollo de la ingeniería industrial, se ha avanzado notablemente en la búsqueda de métodos que permitan aumentar la cantidad y calidad de los productos. Por ello que la pregunta es válida. No obstante, la ergonomía tiene un carácter integrativo y anticipativo, ya que tiende a crear herramientas, máquinas, puestos de trabajo y métodos que se adapten a las capacidades y limitaciones humanas. En otras palabras, cuando se diseña cualquier actividad en que tendrá participación el hombre, es cuando debe evaluarse los efectos que dichos elementos podrían tener sobre quien interactúa con ellos. No hay que olvidar que desde una simple herramienta manual hasta los más complejos sistemas industriales, son creados por seres humanos para ayudarse en el cumplimiento de sus tareas. (Apud, E, Meyer, F. 2003).

La Ergonomía tiene dos grandes ramas: una se refiere a la ergonomía industrial, biomecánica ocupacional, que se concentra en los aspectos físicos del trabajo y capacidades humanas tales como fuerza, postura y repeticiones de movimientos. (Guillén Fonseca, Martha. 2006).

Una segunda rama se refiere a los factores humanos orientada a los aspectos psicológicos del trabajo como la carga mental y la toma de decisiones. (Guillén Fonseca, Martha. 2006). 
En los últimos años se ha dado importancia al estudio del sistema hombre-máquina-ambiente; es decir, la ergonomía. Anteriormente, el diseño de máquinas y ambiente laboral no se tomaba en consideración las destrezas, habilidades y otras características del trabajador, por lo que ocurrían errores excesivos, demoras en la ejecución de trabajo, disminución de la calidad y la productividad. (Torres T, Rodríguez M. 2007).

Esta situación ocurre regularmente en nuestro país. El nivel de productividad en Ecuador, lamentablemente es bajo, siendo uno de los principales obstáculos para ser un país desarrollado e invadir mercados internacionales. (Torres T, Rodríguez M. 2007).

No se conoce que en el Ecuador se hayan realizado estudios relacionados con la ergonomía en las industrias, por lo que se desconoce el grado de incidencia que las condiciones no favorables de trabajo de las personas tengan sobre el nivel de competitividad de las empresas. (Torres $\mathrm{T}$, Rodríguez M. 2007).

\section{Salud Ocupacional}

La Organización Mundial de la Salud la define como una actividad multidisciplinaria que promueve y protege la salud de los trabajadores. Esta disciplina busca controlar los accidentes y las enfermedades mediante la reducción de las condiciones de riesgo.

Los problemas más usuales de los que debe ocuparse la salud ocupacional son las fracturas, cortaduras y distensiones por accidentes laborales, los trastornos por movimientos repetitivos, los problemas de la vista o el oído y las enfermedades causadas por la exposición a sustancias antihigiénicas o radioactivas, por ejemplo. También puede encargarse del estrés causado por el trabajo o por las relaciones laborales. (Definición y objetivos de salud ocupacional y ergonomía. 2013).

Cabe destacar que la salud ocupacional es un tema de importancia para los gobiernos, que deben garantizar el bienestar de los trabajadores y el cumplimiento de las normas en el ámbito del trabajo. Para eso suele realizar inspecciones periódicas, que pretenden determinar las condiciones en las que se desarrollan los distintos tipos de trabajos.

Es importante tener en cuenta que la precariedad del empleo, incide en la salud ocupacional. Una empresa que tiene a sus trabajadores en negro (es decir, que no cuentan con cobertura médica) y 
que presenta un espacio físico inadecuado para el trabajo, pone en riesgo la salud de las personas. (Definición y objetivos de salud ocupacional y ergonomía. 2013)

En realidad, mucho se ha adelantado en el manejo y aplicación de la Ergonomía en diversos campos de las ciencias del trabajo. Sin embargo, poco se había relacionado esta ciencia con los problemas de salud y muy en particular con la relación salud-trabajo. No obstante, en los últimos 20 años una gran cantidad de instituciones e investigadores han dedicado sus esfuerzos a la aplicación de la Ergonomía como un elemento fundamental para la prevención y promoción de salud. (Almirall Hernández J 2014).

El problema de la salud-enfermedad es motivo de interés en cualquier parte del planeta, independientemente del nivel socioeconómico y del desarrollo de los medios de producción. Se convierten los índices de salud en unos de los más usados y fidedignos para evaluar la función de gobierno. (Almirall Hernández J 2014).

Las alteraciones de salud de una población determinada son una expresión de sus condiciones de vida y estas, a su vez, de la forma en que dicho grupo social se inserta en el proceso general de reproducción del conjunto de la sociedad, en un momento histórico concreto y bajo determinadas condiciones naturales.

Ante el desarrollo científico técnico y su expresión en el ámbito de la medicina, han surgido con gran fuerza los términos y procederes de un criterio preventivo, y, como consecuencia, la necesidad de establecer sistemáticamente acciones para la prevención de la salud. (Almirall Hernández J 2014).

En este sentido, numerosos autores han demostrado que las enfermedades están en función del desarrollo social, el cual presenta diferentes grupos o clases que tienen una forma particular de expresar este pro-ceso de salud-enfermedad. (Almirall Hernández J 2014).

En el orden de las ideas anteriores la importancia de la ergonomía en la salud ocupacional, lo primero a tener en cuenta es que desde las distintas compañías se debe promover el bienestar físico, mental y social de cada trabajador. Para llevarlo a cabo, se debe evitar el deterioro del trabajador a causa de las deficientes condiciones de trabajo. Para ello, uno de los tipos es resguardar a los empleados de los peligros que pueden resultar de la exposición a agentes 
nocivos. Por otro lado, se debe cuidar a los trabajadores para que conserven su integridad fisiológica y psicológica. Así se ve que existen riesgos para la postura, para el sistema visual y cardiovascular de las personas y pueden ser el comienzo de enfermedades profesionales. Por eso, la ergonomía se refiere al hecho de ajustar el entorno de vida y trabajo a medida del trabajador en pos de su calidad de vida. Para lograrlo, desde las distintas compañías se debe propiciar la seguridad a través de un ambiente apto para el empleado. Las condiciones de trabajo es un punto crucial para optimizar la salud de los empleados ya que, entre otras cuestiones, al bajar el nivel de estrés y de fatiga física se logrará mayor efectividad en la producción. Así, desde las empresas deben encontrar otras alternativas de trabajo en contraste con el trabajo monótono y automatizado ya que lo único que logran es bajar el rendimiento del personal. (Importancia de la ergonomía en la salud ocupacional. 2012).

Por este motivo, la ergonomía en los puestos de trabajo está en estrecha vinculación ya que la postura, los esfuerzos, el ambiente como así también los medios de señalización son cruciales para resguardar la salud del personal.

En los diferentes puestos de trabajo es de suma utilidad el hecho de observar cuáles son los mayores peligros para tratar de disminuir los problemas del trabajador. Por un lado, los problemas posturales, los malos esfuerzos y los movimientos bruscos son los protagonistas en la jornada laboral de ciertos lugares de trabajo.

En el caso de los problemas posturales, muy frecuente en trabajos con tareas pesadas, así como en empleos de oficina, uno de los consejos es reducir los esfuerzos disminuyendo las cargas y cambiar las posturas evitando permanecer estáticos.

Entre uno de los efectos nocivos de las malas condiciones laborales se puede mencionar el factor psicológico como el estrés, la fatiga y la monotonía. (Importancia de la ergonomía en la salud ocupacional. 2012)

\section{Algunas medidas ergonómicas preventivas}

De los anteriores planteamientos expresados, una de las ramas de la ergonomía es la que tiene en cuenta el aspecto físico, es decir, las posturas más adecuadas para la persona dentro del ámbito laboral. Esta apunta a evitar riesgos, por ejemplo, agacharse con las piernas derechas, girar con el 
cuerpo mientras se tiene peso encima o cargar peso en los hombros. Por el contrario, la ergonomía apela a cambiar hábitos laborales. Es decir, en vez de comportamientos como los anteriormente descriptos, las acciones correctas serían flexionar las rodillas al inclinarse, para que las piernas sean las que hagan fuerza y levantar los objetos hasta el pecho. Además, si el trabajador tiene que levantar una carga excesiva, debería ser ayudado por otro compañero. (Importancia de la ergonomía en la salud ocupacional. 2012).

En cuanto al cuidado de los ojos, es fundamental tener presente que el uso de la computadora en exceso provoca una fatiga visual, al fijar los ojos durante un largo periodo de tiempo provocando ardor como dolores de cabeza. Para que esto no suceda, se debe evitar la opacidad en las imágenes y que la luz sea reflejada en la computadora siendo importante una adecuada iluminación en el lugar de trabajo. (Importancia de la ergonomía en la salud ocupacional. 2012).

Los profesionales del área de la salud atienden trabajadores que, en muchos casos, presentan patologías derivadas de sus condiciones de trabajo. Por ejemplo, en el mundo moderno el estrés laboral, los síntomas músculo-esqueléticos asociados a trabajo repetitivo, posturas inadecuadas y manejo manual de materiales, la obesidad vinculada a trabajo sedentario, la fatiga crónica, etc., podrían disminuir o aminorarse con un adecuado diseño del trabajo. Desde este punto de vista, es importante incluir en la formación de los profesionales de la salud conceptos de ergonomía, ya que, si los agentes causales persisten en el medio ambiente laboral, los tratamientos no serán efectivos y los trabajadores seguirán reiterando síntomas que les alteran su bienestar físico y mental. (Apud E, Meyer F 2003)

Según Montalvo AA, Cortés YM, Rojas MC (2015), en su estudio relacionado los trastornos músculo esqueléticos y los factores de riesgo ergonómicos en personal de enfermería de una clínica en la Costa Atlántica. Sus resultados arrojaron que el dolor de espalda y en mano-muñeca derecha está asociado significativamente $(\mathrm{p}<0,05)$ al riesgo de carga física.

Apud E, Meyer F (2003) informa que de acuerdo a diversos estudios realizados en Europa y Estados Unidos, se estima que entre 50 y $90 \%$ de los usuarios habituales de computadoras sufren fatiga ocular, ojos rojos y secos, tensión de párpados, lagrimeo, sensación de quemazón, visión borrosa y dificultad para enfocar objetos lejanos, al mismo tiempo las posturas corporales inadecuadas que adoptan les generan tensión muscular que se traduce en dolor de cabeza, cuello 
y espalda. También se han reportado casos en los que, debido a estrés, se presenta nerviosismo y hasta mareo. Según datos publicados por el Instituto Nacional de Investigación y Seguridad de Francia, a partir de las cinco horas de trabajo frente a una computadora aumentan los síntomas de problemas visuales, por lo que en un plazo relativamente corto se genera disminución de la productividad y del grado de concentración. En efecto, estos instrumentos llegaron para quedarse y para volver más sencilla nuestra vida, pero si no tenemos cuidado, su uso puede ser contraproducente. No hablamos de historias de ciencia ficción donde robots y computadoras toman el control de la vida en la Tierra, sino que la mayoría de la gente que pasa muchas horas frente a la pantalla puede desarrollar una serie de problemas visuales y dolencias corporales.

Garzon Quintero J D (2009) identifico en su estudio la necesidad de diseñar un conjunto de estrategias a manera de Plan de Mejoramiento, enfocado a tratar temas como: Refuerzo del Programa de Salud Ocupacional de la Empresa, refuerzo del Subprograma de ergonomía, relacionado con los trastornos músculo esquelético y un conjunto de actividades de seguridad industrial dirigidas al personal liniero.

\section{Conclusiones}

Para dar por concluido, la ergonomía y los factores de riesgo en Salud ocupacional deben ser contemplados de forma sistematizada en cada puesto laboral, mediante las revisiones periódicas. Los ambientes adecuados y amigables reducen consecuencias negativas en la salud, mediante el cumplimiento de lo regulado en relación con riesgos laborales en salud ocupacional.

\section{Referencias bibliográficas}

Almirall Hernández J. Ergonomía. (20149. Su aplicación en salud ocupacional. Recuperado de www.sld.cu/galerias/pdf/sitios/insat/cap2.pdf.

Apud, E, Meyer, F. (2003). La importancia de la ergonomía para los profesionales de la salud. Ciencia y enfermería, 9(1), 15-20. Recuperado de https://dx.doi.org/10.4067/S071795532003000100003

Definición y objetivos de salud ocupacional y ergonomía. (2013). Recuperado de http://saludocupacionalergonomiaudea.blogspot.com/p/normal-0-21-false-false-false-es-cox.html 
Guillén F, M. (2006). Ergonomía y la relación con los factores de riesgo en salud ocupacional. Revista Cubana de Enfermería, 22(4) Recuperado de http://scielo.sld.cu/scielo.php?script=sci_arttext\&pid=S0864-

03192006000400008\&lng=es\&tlng=es.

Garzon Quintero J D (2009). Factores de riesgos ergonómicos presentes en los trabajadores de lineas y redes de la empresa electrificadora del Huila S.A. e.s.p zona norte, con sede en la ciudad de Neiva. Trabajo de grado presentado como requisito para optar al título de Profesional en Salud Ocupacional UNIVERSIDAD

Importancia de la ergonomía en la salud ocupacional. (2012). Recuperado de http://www.losrecursoshumanos.com/importancia-de-la-ergonomia-en-la-salud-ocupacional/ Montalvo AA, Cortés YM, Rojas MC. (2015)Riesgo ergonómico asociado a sintomatología musculo esquelética en personal de enfermería. Hacia promoc salud. 2015; 20(2): 132-146. DOI: 10.17151/hpsal..20.2.11.

Torres T, Rodríguez M. (2007). Evaluación Ergonómica de Puestos de Trabajo de la Industria Pesquera del Ecuador. Revista Tecnológica ESPOL, 20(1), 139-142, ISSN : 0257-1749 